\title{
Effect of 4-week High Intensity Training on Immune Function of Weight Lifters
}

\author{
Gao Dongqing \\ Department of physical education, Shanghai University of Engineering Science, Shanghai 201620, China \\ qtryxk@126.com
}

\begin{abstract}
OBJECTIVE: To explore the effect of high intensity training on serum testosterone, cortisol and immune function of weight lifters. 4-week intensive training was carried out. METHODS: The subjects of this experiment are 12 weight lifters. The training loads in the 4 weeks were set respectively around $75 \%$, $80 \%, 80 \%$ and $85 \%$ of maximum heart rate of each subjects, training 5 days and a half each week. Strength training was on Tuesday afternoons and Thursday mornings, movement training in the other sessions. Subjects were training together and instructed by full-time coaches in the whole training process. Blood samples were drawn in the second week, before and after 4-week high intensity training. Serum testosterone, serum cortisol, hemoglobin and the percentage of lymphocyte subpopulations were determined. RESULTS: After 4week high intensity training, $\mathrm{T}$ had a significant decrease compared with that before the training and that in the second week $(\mathrm{P}<0.01)$. $\mathrm{C}$ had a significant increase compared with that before the 4-week high intensity training $(\mathrm{P}<0.01)$ and had no significant change compared with that of the second week $(\mathrm{P}>0.05)$. $\mathrm{T} / \mathrm{C}$ ratio had a significant decrease compared with that before the 4-week high intensity training $(\mathrm{P}<0.01)$. Hemoglobin and CD4 \% had a significant decrease compared with that before the 4-week high intensity training $(\mathrm{P}<0.05) . \mathrm{CD} 8 \%$ had no significant change compared with that in the second week and before the 4-week high intensity training $(\mathrm{P}>0.05)$. $\mathrm{CD} 4 / \mathrm{CD} 8$ ratio had a significant decrease compared with that before the 4-week high intensity training $(\mathrm{P}<0.01)$. CONCLUSION: The high intensity training may result in a significant decrease of $\mathrm{T}, \mathrm{T} / \mathrm{C}$ ratio, $\mathrm{CD} 4, \mathrm{CD} 4 / \mathrm{CD} 8$ ratio and hemoglobin. During sports training, $\mathrm{T} / \mathrm{C}$ and $\mathrm{CD} 4 / \mathrm{CD} 8$ ratio can be used for monitoring athletes' body functions, which is distinctively meaningful for determining and preventing over-fatigue of athletes.

Index Terms - High intensity training, Testosterone; Cortisol, Hemoglobin, Immune function
\end{abstract}

\section{Introduction}

Since 1970's, sports circle has paid attention on the relationship between sports training and male hormone which is represented by blood testosterone and male hormone. Many scholars have studied and believed that a long time exercise might induce a rise followed by a decrease in blood testosterone level, and an exhaustive exercise brings about a decrease in total testosterone, a rise in sex hormone-binding protein level, an extremely low free testosterone level and a rise in plasma corticosterone level. Meanwhile, lots of researches are on the changes of body immune functions after acute exercise of loads. But few researches are reported on the changes of body endocrine immune functions after prolonged intensive training. This paper is based on the experiment on 12 weight lifters, has observed the effect of 4-week heavy-load training on their endocrine immune functions, and discussed their hemoglobin changes and the mechanism of exerciseinduced anemia.

\section{A. Materials}

12 specially recruited weight lifters were the subjects, with the age at $20.2 \pm 1.3$ years, weight $65.5 \pm 1.6 \mathrm{~kg}$, height $162.7 \pm 1.3 \mathrm{~cm}, 3.6 \pm 1.7$ years of training experience. After 2 weeks, 3 of them quit the training due to severe cold, the remaining 9 subjects entered into the final analysis.

\section{B. Methods}

1) Arrangement of training load: 4-week intensive training was carried out. The training loads in the 4 weeks were set respectively around $75 \%, 80 \%, 80 \%$ and $85 \%$ of maximum heart rate of each subjects, training 5 days and a half each week. Strength training was on Tuesday afternoons and Thursday mornings, movement training in the other sessions. Rest on Saturday afternoons and whole Sundays. Subjects were training together and instructed by full-time coaches in the whole training process.

2) Blood sample collecting and processing: $5 \mathrm{ml}$ cubical vein blood was taken before and after the training and on the Sunday of the second week in the morning when the subjects had an empty stomach. Blood routine test had been carried out with the $1 \mathrm{ml}$ of the blood sample after heparin anticoagulation; $\mathrm{T}$ lymph cell subgroup test had been conducted for the percentages of CD4+ and CD8+ with $2 \mathrm{ml}$ blood sample after heparin anticoagulation. The remaining blood sample had immediately been treated centrifugally and the upper layer of serum had been packed and sealed into refrigerators for future use.

3) Indicator test: Measurement of testosterone ( $\mathrm{T})$ test: radioimmunoassay method. Reagent supplier:Diagnostic Systems Laborutories, Inc. USA. Detecting instrument: GC$911 \mathrm{r}$ radioimmunoassay counter.

Measurement of cortisol (C): competition method. Reagent supplier: Bechman Coulter Inc. USA. Detecting instrument: Bechman Coulter Access Immunoassay system (Beckman Coulter Inc, USA).

Measurement of CD4 and CD8: fluorescent double labeling method. Reagent supplier: Bechman Coulter Inc. USA. Detecting instrument: Bechman Coulter Access Immunoassay system(Beckman Coulter Inc,USA).

4) Statistics methods:: SPSS15.0 was employed for data analysis and processing. All data were indicated with the mean \pm standard deviation. Data within groups before, during and 
after the experiment were compared with Paired-Samples $\mathrm{T}$ Test. $\mathrm{P}<0.05$ indicated significant differences, and $\mathrm{P}<0.01$ indicated very significant differences.

\section{Results}

\section{A. Changes of $T, C$ and $H b$ before, during and after high intensity training}

Table I shows as follows: after 4-week high intensity training, the $\mathrm{T}$ level in the 2nd week was higher than that before the training, but there was no significant difference $(\mathrm{P}>0.05)$; T level after the training was significantly lower than that before the training and that in the 2 nd week $(\mathrm{P}<0.01)$. C level after the training had a significant rise than that before the training $(\mathrm{P}<0.01)$, no significant difference was found when compared with that in the 2 nd week $(\mathrm{P}>0.05)$. $\mathrm{T} / \mathrm{C}$ ratio after the training was significantly lower than that before the training, and dropped markedly than that in the 2nd week $(\mathrm{P}>0.05)$. Hb level after the training was evidently lower than that before the training $(\mathrm{P}>0.05)$; compared with that in the 2nd week, no distinct difference was detected $(\mathrm{P}>0.05)$.

TABLE I Comparison of Serum T, $\mathrm{C}$ and $\mathrm{Hb}$ before, during and after the High intensity Training

\begin{tabular}{|c|l|l|l|}
\hline Indicator & Before the Training & The 2nd Week & After the Training \\
\hline $\mathrm{T}(\mathrm{ng} / \mathrm{dl})$ & $683.55 \pm 182.41$ & $697.32 \pm 119.72$ & $621.64 \pm 140.60^{* * \#}$ \\
\hline $\mathrm{C}(\mathrm{ug} / \mathrm{dl})$ & $15.90 \pm 4.64$ & $18.36 \pm 6.13$ & $19.68 \pm 4.40^{* *}$ \\
\hline $\mathrm{T} / \mathrm{C}$ & $42.99 \pm 15.13$ & $37.98 \pm 11.67^{*}$ & $31.59 \pm 13.22^{* * *}$ \\
\hline $\mathrm{Hb}(\mathrm{g} / \mathrm{L})$ & $157.56 \pm 2.56$ & $153.86 \pm 3.15$ & $146.276 \pm 4.61^{*}$ \\
\hline
\end{tabular}

Compared with that before the training, ${ }^{\sharp} P<0.05,{ }^{\#} P<0.01$; Compared with the 2nd week, ${ }^{\#} P<0.05,{ }^{\#} P<0.01$.

\section{B. Changes of CD4, CD8 and CD4/CD8 before, during and after high intensity training}

Table II shows as follows: after 4-week high intensity training, CD4 level was evidently lower than that before the training $(\mathrm{P}>0.05)$. There was no distinct difference between $\mathrm{CD} 8$ level after and before the training, or between that before the training and in the 2nd week $(\mathrm{P}>0.05)$. CD4/CD8 ratio after the training was significantly lower than that before the training $(\mathrm{P}<0.01)$, and visibly lower than that in the 2 nd week $(\mathrm{P}<0.05)$.

TABLE II Comparison of CD4, CD8 and CD4/CD8 before, during and after the High intensity Training

\begin{tabular}{|c|l|l|l|}
\hline Indicator & Before the Training & The 2nd Week & After the Training \\
\hline CD4 (\%) & $33.5 \pm 2.3$ & $32.1 \pm 3.9$ & $30.6 \pm 2.7^{*}$ \\
\hline CD8 (\%) & $27.1 \pm 1.4$ & $28.7 \pm 1.3$ & $27.8 \pm 2.5$ \\
\hline CD4 / CD8 & $1.24 \pm 0.07$ & $1.12 \pm 0.09^{*}$ & $1.10 \pm 0.07^{* *}$ \\
\hline
\end{tabular}

Compared with that before the training, \# $\mathrm{P}<0.05$, \# \# $\mathrm{P}<0.01$; Compared with the 2nd week, \#P<0.05, \# \# $\mathrm{P}<0.01$

\section{Discussions}

\section{A. The Effect of High intensity Training on Testosterone and Cortisol}

Testosterone is one of the main hormones promoting constructive metabolism. Changes of testosterone are somewhat regular. Sports and emergencies arouse visible change of testosterone. Sports-induced changes are mainly influenced by factors like exercise density, load intensity, load, duration and so on. Generally speaking, exercises first cause a rise in blood testosterone which comes to a peak as the exercise prolonged and then goes down. If the exercise load continually increases, organism shows no adaptation, blood testosterone will be declining to a level lower than that before exercising. This study observed that, after 4-week high intensity training, the subjects' testosterone level dropped sharply. As to the rise of testosterone in the early stage of exercises, we believed it is resulted from the temporary adaptation of body's endocrine function, when exercise intensity and duration increase, endocrine function will be disordered and induce the decrease of testosterone level.

The biological roles of cortisol are mainly to participate in metabolism, maintain normal glucose metabolism in the body, and keep blood sugar levels stable. Adrenal cortex function of athletes is stronger than ordinary people. But excessive sports training will cause a decline of adrenal cortex function, and then a distinct decrease of physical strength. Literature reports showed that, immediately after a short, low intensity exercise, or a high intensity but long intermittent one, plasma cortisol, is generally unchanged or declined to some extend. In this kind of sport, adrenocorticotropic hormone experiences an immediate increase, while cortisol comes to a peak after 10 minutes. From medium intensive sports to high intensive sports, as the intensity increases, plasma cortisol density goes up, which is to regulate the body's ability to adapt to outside stimuli $[1,2,3]$.

This study found that after the 4-week training, the subjects' cortisol levels significantly increased. Exercise stress facilitates an increase in cortisol secretion and promote glucose metabolism, so as to meet the increase of function loads and energy consumption while organisms are in movement. Long-term training inhibits the hypothalamus pituitary - adrenal function of ordinary people, making the density of plasma cortisol levels decrease significantly, thus affecting the athletic capacity. While for athletes, the hypothalamus - pituitary - adrenal function suppression is delayed, and is restrained to a relatively low degree, which helps athletes to maintain a good state of exercising, to resist fatigue. However, in prolonged high-intensity exercises, the consumption of body's glycogen is very large, and results in the decomposition of protein into amino acids to perform gluconeogenesis. At this moment, the hypothalamus - pituitary - adrenal axis function is weakened, adrenal cortex hormone secretion reduces, and thus the level of serum cortisol is low.

Many scholars believe that testosterone/cortisol ratio helps to understand the balance of body anabolic and catabolic 
states. The ratio has been recognized currently as a sensitive indicator for the assessing and monitoring of overtraining, and fatigue recovery[4,5]. It is generally accepted that high ratio means functions in a good state, and adapt to exercise loads. The ratio comes down when the body is in fatigue or not suit to the load. It is found in the study that after the 4-week high intensity training, the subjects' testosterone/cortisol ratio decreased evidently. The reason of the decrease was the changes of the secretion level of testosterone and cortisol in the body after high intensity training. Large number of studies further confirmed that after a period of training, the enhancement of athletes' athletic ability was always accompanied by an increase in the testosterone/cortisol ratio; while after a continual high-intensity training, athletes suffered fatigue accumulation, blood testosterone kept on declining, cortisol recovery was also in a relatively high level of failure, and this was when the testosterone/cortisol ratio decreased significantly.

\section{B. The Effect of High intensity Training on CD4 and CD8}

The effect of sports training on CD4 and CD8 are related to the nature of training load (long-term training, one-time acute sport), intensity (appropriate intensity, high intensity) and the subjects' athletic ability (athletes, ordinary people). Nieman observed a 4-week intensive training of seven middledistance runners. In the observation, after 36 hours of rest, CD4T cells were in a downward trend, CD8T cell percentage was significantly increasing, and the $\mathrm{CD} 4 / \mathrm{CD} 8$ ratio decreased[6]. Macneil observed cyclists that after 4-week high-intensity training, the CD8T cell ratio in peripheral blood was significantly higher, while the CD4/CD8 ratio decreased significantly in comparison with that before the training, and was visibly lower than that in the 2 nd week. The ratio of CD4/CD8 reflects the overall immune state of the body. Favorable sports training promotes the CD4/CD8 ratio significantly, enhance the immunity level of the body. The inverted ratio of CD4/CD8 in the athletes after high intensive training indicated that long term high intensive training may affect the immune level of the body and result in the immune dysfunction.

\section{The Effect of High intensity Training on hemoglobin}

Researches have proved that exercises may cause changes in hemoglobin level, and the degree of change is related to the nature, intensity and duration of an exercise. Mi Jue stated that hemoglobin was a sensitive indicator to reflect the appropriateness of training load[7]. In the first two weeks of training in this study, there was no visible change in hemoglobin level, but it dropped distinctively after the whole training. This further confirmed the close relationship between exercise intensity and hemoglobin. Factors for the decrease of hemoglobin level in high intensity training are as follows. First, the auto-oxidation rate of hemoglobin in red blood cells accelerates, oxidizes the lipid of red cell membrane, reduces its deformability, and increases its fragility. Therefore, strong blood flow leads to the breakdown of some red blood cells and the decomposition of hemoglobin. Secondly, heavy sweating in exercises excrete iron element out of the body, and causes iron-deficiency-induced hemoglobin regeneration deficiency, which leads to hemoglobin decline. Thirdly, the mechanical role of the exercise suppresses some parts of the body, resulting in mechanical injury of red blood cells in local blood vessels, which causes hemoglobinuria and lead to the decrease of hemoglobin level.

\section{Conclusion}

After 4-week high intensity training, testosterone, ratio of testosterone/cortisol, CD4, ratio of CD4/CD8, and hemoglobin dropped markedly, which appeared as inadequate adaptation to the training load, and fatigue of different degrees. The study showed that, prolonged sports training and prolonged intensive training may restrain the endocrine immune function. In athletes' high intensity training, ratio of testosterone/cortisol, and ratio of $\mathrm{CD} 4 / \mathrm{CD} 8$ can be employed jointly to monitor the athlete's function changes. This is significant for determining and preventing athletes' over-fatigue.

\section{References}

[1] Tvede N. Mechanisms of B lymphocyte suppression induced by acute physical exercise . J Clin lab immunol, 1999, 30(7):169-173.

[2] Nieman DC, Nehlsen SL. The effect of acute and chronic exercise on immunoglobulins . Sports Medicine, 2005, 79(11):183-201.

[3] Bente KP, Anders DT. Effects of exercise on lymphocytes and cytokines . Br J Sports Med, 2008, 112(34):246-251

[4] Xie Minhao, Feng Yiquan, Yang Tianle, et al. Blood Testosterone and Sports . Sports Science, 1999,19(2):80-83.

[5] Quan Yefei, Status Quo of Domestic Research on Physical Roles of Male Hormone and the Relevant Factors . Sports Science, 1999, 19(3):66-68.

[6] Nieman DC, Berk LS, Westerberg M, et al. Effects of long-endurance running on immune system parameters and lymphocyte function in experienced marathoners .Int Sports Med, 2003,49(10):317-323.

[7] Mi Jue. Discussion on the Relationship between Hemoglobin Level and the Intensity of Pre-match Training . Journal of Nanjing Sport Institute, 1999, 13(1):54-56. 